\title{
Analytical Study on Pulsed-Laser Processing for Acrylonitrile Butadiene Styrene/PolyVinyl Chloride
}

\author{
Chang-Wei XIONG ${ }^{1}$, Ching-Yen HO ${ }^{1,2,3}{ }^{*}$, Dongkai QIAO ${ }^{2}$ \\ ${ }^{1}$ Department of Mechanical and Electrical Engineering, Dongguan Polytechnic, Dongguan 523808, Guangdong, China \\ ${ }^{2}$ School of Mechanical and Electrical Engineering, Guangdong University of Petrochemical Technology, Maoming \\ 525000, Guangdong, China \\ ${ }^{3}$ Department of Mechanical Engineering, Hwa Hsia University of Technology, Taipei 235, Taiwan, R.O.C.
}

cross ref $h$ ttp://dx.doi.org/10.5755/j01.ms.26.1.20310

Received 07 March 2018; accepted 31 September 2018

\begin{abstract}
This study developed a model involving the energy balance and decomposition at the ablated interface due to the heat transport, optical decomposition and activation energy in Acrylonitrile Butadiene Styrene/PolyVinyl Chloride (ABS/PVC). The prediction for ablation depth obtained from this study is compared with the available experimental data. The predicted results agree with the measured data. The effects of laser processing parameters, optical and thermal properties of $\mathrm{ABS} / \mathrm{PVC}$ on the variation of the laser-ablated depth with the laser fluences are also discussed. The results validate that the pulsed-laser ablation rate at the initial short period is dominated by optical penetration absorption for laser and is linearly proportional to the logarithm of the laser fluence by a ratio factor "optical absorption length" (i.e., the ablation rate follows Beer's law at the initial short period).

Keywords: pulsed-laser, depth, processing, ABS/PVC.
\end{abstract}

\section{INTRODUCTION}

Laser has many applications such as welding [1], microstructuring [2-5] and ablating [6]. Laser is a wellestablished tool for polymer processing [7] due to different machining characteristics from the traditional processing technology. Pulsed-lasers (picosecond and femtosecond) are able to obtain microstructures with high precision. In the process, the heat-affected zone is small and the melt produced also approaches a minor region. Consequently, the laser radiation can directly etch the polymer material and increase the accuracy of the topographical features generated on the substrate surface. This technology represents a powerful technique for microstructure, allows a direct and fast processing of a wide variety of polymer substrates and geometries, and includes the microfabrication of complex structures on flat and non-flat surfaces [8].

Ablation mechanism of laser for plastics is not the same as that of ceramics [9-12] and metal [13-17]. The laser ablation for metal material is induced by vaporisation and melting. Most plastics tend to decompose below the evaporation temperature and long chains of molecules are decomposed into fragments before they are removed by laser from bulk material. The ablated depth per pulse mainly depends on the amount of broken bonds in polymeric chain $[18,19]$. The laser heating spot inflates rapidly. This quick expansion leads to volume explosion which is the force for expelling of the material.

Water resistance, acid and alkali resistance, chemical resistance of polyvinyl chloride (PVC) are very good and the price of PVC is cheap. The disadvantage is that the production technology is high and there are special

\footnotetext{
* Corresponding author. Tel.: +86-13423291876.

E-mail address: 2563794452@qq.com (C.Y.Ho)
}

requirements for the mold or screw. Acrylonitrile Butadiene Styrene (ABS) has good toughness and strength, good fluidity and easy molding, but the disadvantage of $\mathrm{ABS}$ is that it is expensive and has poor aging resistance. It starts to change color after one month. Therefore, Acrylonitrile Butadiene Styrene/ PolyVinyl Chloride (ABS/PVC) is usually used to fabricate various products instead of ABS or PVC, which possesses the advantages of $\mathrm{PVC}$ and $\mathrm{ABS}$. The price of $\mathrm{ABS} / \mathrm{PVC}$ is also moderate and relatively cheap. The models of refs. [9-17] were mainly used to investigate the laser ablation of metals and ceramics. However, the similar model used in this paper focuses on the study of laser ablation for polymer.

This study develops a model to predict the ablated depth per pulse for picosecond laser processing of ABS/PVC. The model developed by this work takes the optical and thermal properties of ABS/PVC, decomposition and energy balance at the ablated interface, and the parameters of laser processing into account. The prediction for ablation depth obtained from this study is compared with the experimental data. The effects of laser processing parameters, optical and thermal properties of $\mathrm{ABS} / \mathrm{PVC}$ on the variation of the laser-ablated depth with the laser fluences are also discussed.

\section{THEORETICAL MODEL}

\subsection{Analysis}

Heat transfer equation of pulsed-laser processing for $\mathrm{ABS} / \mathrm{PVC}$ may be written as

$c \rho \frac{\partial T}{\partial t}=k \frac{\partial^{2} T}{\partial z^{2}}+\frac{3 Q}{\pi \delta \sigma^{2}}(1-R) \exp \left[-\left(\frac{t}{\tau_{0}}\right)^{2}-\frac{z}{\delta}-3\left(\frac{r}{\sigma}\right)^{2}\right]$

where $T$ is the temperature; $k$ represents the thermal conductivity; $\rho$ is the mass density; $c$ denotes the heat 
capacity; $z$ signifies the spatial coordinate vertically toward the interior of the workpiece; $t$ stands for time. A 60ps UV pulsed-laser with a wavelength $266 \mathrm{~nm}$ is utilized to conduct this laser ablation of ABS/PVC, which mode is $\mathrm{TEM}_{00}$ with the profile of Gaussian distribution. The symbols $Q, R, \delta, \tau_{0}$ and $\sigma$ correspondingly represent the laser power, reflectivity, optical absorption length, pulse duration at FWHM and energy-distribution radius. The energy-distribution radius denotes the range of energydistribution for laser beam. 3 in Eq. 1 are taken to assure 90 percent of laser energy included within the energydistribution radius.

The temperature is assumed to be the ambient temperature at the initial condition and boundary condition of $z=\infty$. The boundary condition on the surface of workpiece is taken as

$-k \frac{\partial T(0, t)}{\partial z}+h\left[T(0, t)-T_{\infty}\right]=0$

where $T_{\infty}$ is the ambient temperature; $h$ is the heat convection coefficient.

The energy balance equation at the decomposition interface is

$-k \frac{\partial T}{\partial z}=\rho \gamma \frac{\partial f}{\partial t}, \quad T(f, t)=T_{d} ;$

where $T_{\mathrm{d}}$ is the decomposition temperature of polymer; $\gamma$ is the thermal activation energy which is related to bond energy of polymers; $f$ is the decomposition interface.

The nondimensional parameters are defined as

$\theta=\frac{T-T_{\infty}}{T_{d}-T_{\infty}}, \quad \tau 0 L=\frac{\tau_{0}}{\frac{c L^{2}}{k}}=\frac{\tau_{0}}{\tau L}, \quad \xi=\frac{z}{L}, \quad \tau=\frac{t}{\tau_{0}}, \quad H=\frac{h L}{k}$,

$F=\frac{f}{L}, \quad R=\frac{r}{L}$

where $L$ is the thickness of workpiece. Therefore, the nondimensional governing equation yields

$\frac{\partial \theta}{\partial \tau}=\tau 0 L \times \frac{\partial^{2} \theta}{\partial \xi^{2}}+\frac{J}{\delta c\left(T_{d}-T_{\infty}\right)}(1-R) \exp \left[-\tau^{2}-\frac{L \xi}{\delta}-3\left(\frac{L R}{\sigma}\right)^{2}\right] ;$

where laser fluence $J$ equals to $3 Q \tau_{0} /\left(\pi \sigma^{2}\right)$ and the nondimensional boundary condition on the surface of workpiece is

$-\frac{\partial \theta(0, \tau)}{\partial \xi}+H \theta(0, \tau)=0$.

The nondimensional form for energy balance equation at the decomposition interface yields

$-\frac{\partial \theta}{\partial \xi}=\Omega_{2} \frac{\partial F}{\partial \tau}, \quad T(F, \tau)=1 ;$

where

$\Omega_{2}=\frac{\rho \varkappa^{2}}{k\left(T_{d}-T_{\infty}\right) \tau_{0}}$.

In order to understand the effects of direct laser optical absorption and thermal diffusion on the temperature and the ablated depth, the Laplace transform is utilized to get the solution. If the Laplace transform of the temperature $\theta$ is symbolized by $\Theta$, the governing equation in Laplace domain is

$$
\begin{aligned}
& s \Theta=\tau 0 L \times \frac{d^{2} \Theta}{\partial \xi^{2}}+\frac{J}{\delta c\left(T_{e}-T_{\infty}\right)}(1-R) \exp \left[-\frac{L \xi}{\delta}-3\left(\frac{L R}{\sigma}\right)^{2}\right] . \\
& \frac{\sqrt{\pi}}{2} e^{\frac{s^{2}}{4}} \operatorname{erfc}\left(\frac{s}{2}\right)
\end{aligned}
$$

and in the Laplace domain, the nondimensional boundary condition on the surface of workpiece is

$-\frac{\partial \Theta(0, s)}{\partial \xi}+H \Theta(0, s)=0$

Let the symbol $\Gamma$ represent the Laplace transform of the nondimensional decomposition interface $F$. The Laplace transform of the energy balance equation is

$-\frac{\partial \Theta}{\partial \xi}=\Omega_{2} s \Gamma, \quad \Theta(\Gamma, s)=\frac{1}{s}$.

\subsection{Solution}

In Laplace domain, the general solution with the initial condition is

$$
\begin{aligned}
& \Theta=c_{1} \times e^{-\sqrt{\frac{s}{\tau 0 L}} \zeta}+c_{2} \times e^{\sqrt{\frac{s}{\tau 0 L} \zeta}}- \\
& \frac{J}{\delta c\left(T_{e}-T_{\infty}\right)}(1-R) e^{-\frac{L \xi}{\delta}-3\left(\frac{L R}{\sigma}\right)^{2}} \frac{\sqrt{\pi}}{2} e^{\frac{s^{2}}{4}} \operatorname{erfc}\left(\frac{s}{2}\right) . \\
& \left(\tau 0 L \times \frac{L^{2}}{\delta^{2}}-s\right)
\end{aligned}
$$

The constant $c_{2}$ is taken to be zero because the temperature is finite at $\xi \rightarrow \infty$. Using the boundary condition on the workpiece surface, we get the constant $c_{1}$

$$
c_{1}=\frac{\left(-H+\frac{L}{\delta}\right)}{\left(H-\sqrt{\frac{s}{\tau 0 L}}\right)} \times \frac{\frac{-J}{\delta c\left(T_{e}-T_{\infty}\right)}(1-R) e^{-3\left(\frac{L R}{\sigma}\right)^{2}} \frac{\sqrt{\pi}}{2} e^{\frac{s^{2}}{4}} \operatorname{erfc}\left(\frac{s}{2}\right)}{\left(\tau 0 L \times \frac{L^{2}}{\delta^{2}}-s\right)} .
$$

Therefore,

$\Theta=\Omega_{1} \frac{e^{\frac{s^{2}}{4}} \operatorname{erfc}\left(\frac{s}{2}\right)}{\left(\tau 0 L \times \frac{L^{2}}{\delta^{2}}-s\right)} \times\left(\frac{H-\frac{L}{\delta}}{H-\sqrt{\frac{s}{\tau 0 L}}} e^{-\sqrt{\frac{s}{\tau 0 L} \xi}}-e^{-\frac{L \xi}{\delta}}\right) ;$

where

$\Omega_{1}=\frac{J(1-R) \sqrt{\pi} e^{-3\left(\frac{L R}{\sigma}\right)^{2}}}{2 \delta c\left(T_{d}-T_{\infty}\right)}$.

The contribution of thermal diffusion to the temperature is the first term in the right-handed side of Eq. 14. However, the contribution of direct laser optical absorption to the temperature is the second term in the right-handed side of Eq. 14.

The nondimensional temperature in time domain is derived by the inverse of Laplace transform. 


$$
\begin{aligned}
& \theta=\Omega_{1}\left\{\sqrt{\tau_{0 L}}\left(H-\frac{L}{\delta}\right) e^{\left(\frac{\tau_{0 L} L^{2}}{2 \delta^{2}}\right)^{2}}\right. \\
& \int_{0}^{\tau}\left[\frac{1}{\sqrt{\pi \tau}} e^{-\frac{\xi^{2}}{4 u \tau_{0 L}}}+\left(\sqrt{\tau_{0 L}} H\right) e^{-H \xi} e^{\tau_{0 L} H^{2} u}\right] e^{\frac{\tau_{0 L} L^{2}(\tau-u)}{\delta^{2}}} \\
& {\left[\operatorname{erf}\left(\tau-u+\frac{\tau_{0 L} L^{2}}{2 \delta^{2}}\right)-\operatorname{erf}\left(\frac{\tau_{0 L} L^{2}}{2 \delta^{2}}\right)\right] d u+} \\
& \left.e^{\left(\frac{\tau_{0 L} L^{2}}{2 \delta^{2}}\right)^{2}+\frac{\tau_{0 L} L^{2} \tau}{\delta^{2}}} e^{-\frac{L \xi}{\delta}}\left[\operatorname{erf}\left(\tau+\frac{\tau_{0 L} L^{2}}{2 \delta^{2}}\right)-\operatorname{erf}\left(\frac{\tau_{0 L} L^{2}}{2 \delta^{2}}\right)\right]\right\}
\end{aligned}
$$

Substituting Eq. 16 into the energy balance equation at the decomposition interface, we get the ablated depth.

$$
\begin{aligned}
& \Omega_{1}\left\{\sqrt{\tau_{0 L}}\left(H-\frac{L}{\delta}\right) e^{\left(\frac{\tau_{0 L} L^{2}}{2 \delta^{2}}\right)^{2}}\right. \\
& \int_{0}^{\tau}\left[\frac{1}{\sqrt{\pi \tau}}\left(\frac{F}{2 u \tau_{0 L}}\right) e^{-\frac{F^{2}}{4 u \tau_{0 L}}}+\left(\sqrt{\tau_{0 L}} H^{2}\right) e^{-H F} e^{\tau_{0 L} H^{2} u}\right] e^{\frac{\tau_{0 L} L^{2}(\tau-u)}{\delta^{2}}} . \\
& {\left[\operatorname{erf}\left(\tau-u+\frac{\tau_{0 L} L^{2}}{2 \delta^{2}}\right)-\operatorname{erf}\left(\frac{\tau_{0 L} L^{2}}{2 \delta^{2}}\right)\right] d u+e^{\left(\frac{\tau_{0 L} L^{2}}{2 \delta^{2}}\right)^{2}+\frac{\tau_{0 L} L^{2} \tau}{\delta^{2}}}} \\
& \left.\left(\frac{L}{\delta}\right) e^{-\frac{L F}{\delta}}\left[\operatorname{erf}\left(\tau+\frac{\tau_{0 L} L^{2}}{2 \delta^{2}}\right)-\operatorname{erf}\left(\frac{\tau_{0 L} L^{2}}{2 \delta^{2}}\right)\right]\right\}=\Omega_{2} \frac{\partial F}{\partial \tau}
\end{aligned}
$$

Combining Eq. 14 with Eq. 11 of Laplace transform of the energy balance equation, one get

$$
\Omega_{1} \times \frac{e^{\frac{s^{2}}{4}} \operatorname{erfc}\left(\frac{s}{2}\right)}{\left(\tau 0 L \times \frac{L^{2}}{\delta^{2}}-s\right)} \times\left(\sqrt{\frac{s}{\tau 0 L}} \times \frac{H-\frac{L}{\delta}}{H-\sqrt{\frac{s}{\tau 0 L}}} e^{-\sqrt{\frac{s}{\tau 0 L}} \Gamma}-\frac{L}{\delta} e^{-\frac{L \Gamma}{\delta}}\right)=\Omega_{2} s \Gamma .
$$

After taking the logarithm for Eq. 18 and rearranging the formula, we obtain the relationship between the laser fluence and the ablated depth in Laplace domain.

$$
\log (J)=-\log \left[\frac{k \times \tau 0 \times(1-R) \sqrt{\pi} e^{-3\left(\frac{r}{\sigma}\right)^{2}} e^{\frac{s^{2}}{4}} \operatorname{erfc}\left(\frac{s}{2}\right)}{2 \delta c \rho \mathcal{L}^{2} \times\left(\tau 0 L \times \frac{L^{2}}{\delta^{2}}-s\right)} \times .\right.
$$

$\left.\left(\sqrt{\frac{s}{\tau 0 L}} \times \frac{H-\frac{L}{\delta}}{H-\sqrt{\frac{s}{\tau 0 L}}} e^{-\sqrt{\frac{s}{\tau 0 L} \Gamma}}-\frac{L}{\delta} e^{-\frac{L \Gamma}{\delta}}\right)\right]+\log [s \Gamma]$

\section{RESULTS AND DISCUSSION}

The variation of ablation rate with the laser fluence was calculated using the Eq. 19, the energy balance at decomposition interface, boundary conditions and initial condition. Table 1 and Table 2 , respectively, list material properties [20] that are used in the theoretical calculation for this model and the parameters that are used in experiments [19] and calculations. While detailed information about the content of PVC in ABS is not provided in [19], the input values of material properties of ABS/PVC matching the experimental data in this simulation are between material properties of ABS and PVC. Therefore, the non-mentioned content of PVC in ABS in [19] can be inversely predicted from this theoretical calculation. Table 1 lists the input values of material properties of $\mathrm{ABS} / \mathrm{PVC}$ for this theoretical calculation, material properties of ABS and PVC.

Table 1. Typical material properties used in this calculation for $\mathrm{ABS} / \mathrm{PVC}$ as well as material properties of ABS and PVC [20]

\begin{tabular}{|l|l|l|l|}
\hline & $\begin{array}{l}\text { Typical material } \\
\text { properties used } \\
\text { in this } \\
\text { calculation-for } \\
\text { ABS/PVC }\end{array}$ & ABS & PVC \\
\hline$R$ & 0.8 & 0.8 & 0.75 \\
\hline$\delta$ & $16.7 \mu \mathrm{m}$ & $20 \mu \mathrm{m}$ & $15 \mu \mathrm{m}$ \\
\hline$\rho$ & $1100 \mathrm{~kg} / \mathrm{m}^{3}$ & $990 \mathrm{~kg} / \mathrm{m}^{3}$ & $1350 \mathrm{~kg} / \mathrm{m}^{3}$ \\
\hline$c$ & $1330 \mathrm{~J} / \mathrm{kgK}$ & $1424 \mathrm{~J} / \mathrm{kgK}$ & $1047 \mathrm{~J} / \mathrm{kgK}$ \\
\hline$\gamma$ & $1 \mathrm{eV}$ & $0.72 \mathrm{eV}[19]$ & $1.76 \mathrm{eV}[19]$ \\
\hline$k$ & $3 \mathrm{~W} / \mathrm{mK}$ & $3.4 \mathrm{~W} / \mathrm{mK}$ & $2.5 \mathrm{~W} / \mathrm{mK}$ \\
\hline$T_{d}$ & $270{ }^{\circ} \mathrm{C}$ & $350{ }^{\circ} \mathrm{C}[19]$ & $200{ }^{\circ} \mathrm{C}[19]$ \\
\hline
\end{tabular}

The value with asterix symbol in parenthesis of Table 2 represents different parametric value used in this calculations from experiments. Although the spot diameter used in this calculation is different from that of experiment, the effect of spot diameter on the ablated depth is not obvious. It can be found from Fig. 2 that the energydistribution radius changed from $10 \mu \mathrm{m}$ to $20 \mu \mathrm{m}$ only gives insignificantly deviation of the ablated depth from experimental data. Besides, the optical absorption length used in this calculation is within the range of experimental values. In Fig. 1, the predicted relation of the ablation rate to laser fluence by this model was shown and compared with the measured data [19]. From this figure, it is also found that the ablation rate increases more slowly at low laser fluences. However, at high laser fluence the ablation rate increases more quickly. Generally, it was recognized that the ablation rate is dominated by the effective absorption length and the ablation threshold according to the Beer's model. The solid line represents the ablation rate calculated by this model and the triangle symbols are the measured data. It is also indicated in Fig. 1 that the calculated results agree well with the measured data.

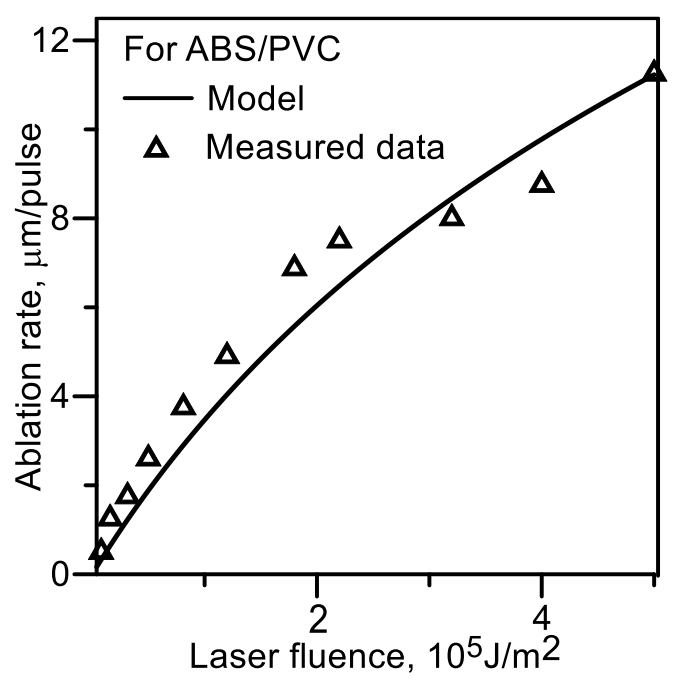

Fig. 1. Comparisons of theoretical and measured ablation rate [19] ABS/PVC for different laser fluences

The integral term at the left-handed side of Eq. 17 is 
from the thermal diffusion. The other terms are attributed to the direct laser fluence. In this study, the order of the thermal and optical properties is $T_{\mathrm{d}} \sim 10^{2 \circ} \mathrm{C}, c \sim 10^{6} \mathrm{Jm}^{-}$ ${ }^{3 \circ} \mathrm{C}^{-1}, \quad L \sim 10^{-3} \mathrm{~m}, \quad \tau_{0} \sim 10^{-12} \quad \mathrm{sec}, \quad k \sim 10^{0} \quad \mathrm{Wm}^{-1} \mathrm{C}^{-1}$, $h \sim 10^{2} \mathrm{Wm}^{-2 \circ} \mathrm{C}^{-1}, \rho \sim 10^{3} \mathrm{kgm}^{-3}, \delta \sim 10^{-6} \mathrm{~m}$. Therefore, the orders of the nondimensional parameters are $\tau_{0 \mathrm{~L}} \sim 10^{-12}, \frac{L}{\delta}$ $\sim 10^{3}$. In Eq. 18, $e^{-\sqrt{\frac{s}{\tau 0 L}} \Gamma}$ of the thermal diffusion term decays more quickly than $e^{-\frac{L \Gamma}{\delta}}$ of the laser absorption term at the initial processing time $(\mathrm{s}>>1)$. This results in it that the effect of direct laser fluence on the ablation rate is far larger than the thermal diffusion at the initial processing time. Finally, the Eq. 17 is simplified as

$$
\begin{aligned}
& \Omega_{1}\left\{e^{\left(\frac{\tau_{0} L^{2}}{2 \delta^{2}}\right)^{2}+\frac{\tau_{0 L} L^{2} \tau}{\delta^{2}}}\left(\frac{L}{\delta}\right) e^{-\frac{L F}{\delta}}\left[\operatorname{erf}\left(\tau+\frac{\tau_{0 L} L^{2}}{2 \delta^{2}}\right)-\operatorname{erf}\left(\frac{\tau_{0 L} L^{2}}{2 \delta^{2}}\right)\right]\right\} \\
& =\Omega_{2} \frac{\partial F}{\partial \tau}
\end{aligned}
$$

Table 2. Laser-processing parameters used in experiments [19] and calculations of this model. (*Different parametric values used in this calculations from experiments)

\begin{tabular}{|l|l|}
\hline & $\begin{array}{l}\text { Laser-processing parameters in } \\
\text { experiments [19] and this } \\
\text { calculations }\end{array}$ \\
\hline Laser wavelength & $266 \mathrm{~nm}$ \\
\hline Laser pulse duration & $60 \mathrm{ps}$ \\
\hline Thickness & $3.22 \mathrm{~mm}$ \\
\hline Laser fluences & $4000 \sim 500000 \mathrm{~J} / \mathrm{m}^{2}$ \\
\hline Spot diameter & $25 \mu \mathrm{m}\left(20 \mu \mathrm{m}^{*}\right)$ \\
\hline Decomposition temperature & $270^{\circ} \mathrm{C}$ \\
\hline Thermal activation energy & $1 \mathrm{eV}$ \\
\hline Optical absorption length & $2-25 \mu \mathrm{m}\left(16.7 \mu \mathrm{m}^{*}\right)$ \\
\hline
\end{tabular}

Integrating Eq. 20, we get

$$
\frac{-\frac{J(1-R) \sqrt{\pi} e^{-3\left(\frac{L R}{\sigma}\right)^{2}}}{2 \delta c\left(T_{d}-T_{\infty}\right)}}{\Omega_{2}}
$$

$\int_{\tau_{e}}^{\tau}\left\{e^{\left(\frac{\tau_{0 L} L^{2}}{2 \delta^{2}}\right)^{2}+\frac{\tau_{0 L} L^{2} \tau}{\delta^{2}}}\left[\operatorname{erf}\left(\tau+\frac{\tau_{0 L} L^{2}}{2 \delta^{2}}\right)-\operatorname{erf}\left(\frac{\tau_{0 L} L^{2}}{2 \delta^{2}}\right)\right]\right\} d \tau+1=e^{-\frac{L F}{\delta}}$

After taking the logarithm of two side of Eq. 21, the relationship between the laser fluence and the ablation depth is obtained.

$$
\begin{aligned}
& \log _{e} J+\log _{e}\left(\frac{-\frac{(1-R) \sqrt{\pi} e^{-3\left(\frac{L R}{\sigma}\right)^{2}}}{2 \delta c\left(T_{d}-T_{\infty}\right)}}{\Omega_{2}}\right. \\
& \int_{\tau_{e}}^{\tau}\left\{e^{\left(\frac{\tau_{0 L} L^{2}}{2 \delta^{2}}\right)^{2}+\frac{\tau_{0 L} L^{2} \tau}{\delta^{2}}}\left[\operatorname{erf}\left(\tau+\frac{\tau_{0 L} L^{2}}{2 \delta^{2}}\right)-\operatorname{erf}\left(\frac{\tau_{0 L} L^{2}}{2 \delta^{2}}\right)\right]\right\} d \tau \\
& +1)=\frac{L F}{\delta}
\end{aligned}
$$

From Eq. 22, it is found that the ablation rate is proportional to the logarithm of the laser fluence by a ratio factor . This is consistent with the Beer's law.

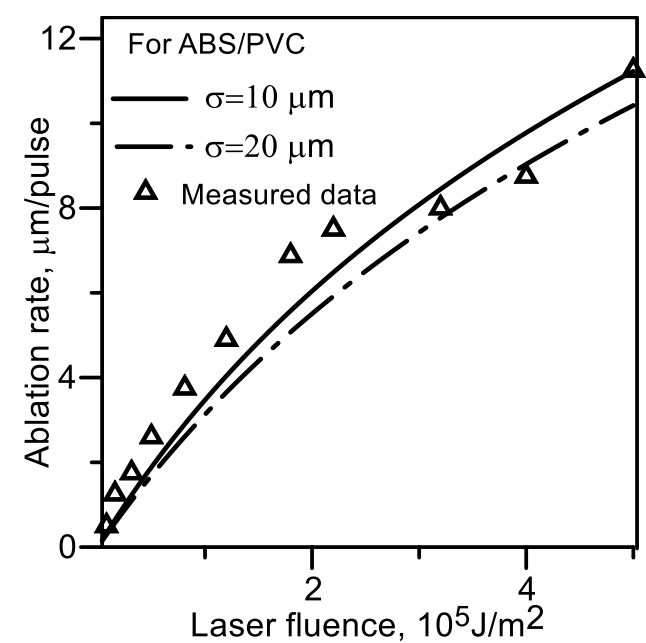

Fig. 2. Relation between ablation depth per pulse and laser fluences for different energy-distribution radii of laser beam

It is shown in Fig. 2 that the ablation depth of laser processing for ABS/PVC varies with the laser fluences at different energy-distribution radii. The wide energydistribution radius of laser beam produces the shallow ablation for different laser fluences because the laser intensity is lower for larger different energy-distribution radius.

Fig. 3 shows how the thermal conductivity affects the variation of the ablation depth per pulse with laser fluences. The solid and dashed lines, respectively, represent the thermal conductivities 3 and $4 \mathrm{~W} / \mathrm{mK}$. The high thermal conductivity leads to fast heat diffusion. This reason induces deep heat penetration which gives deep ablation. Compared with the experimental data as the triangle symbols in Fig. 3, the solid and dashed lines, respectively, agree with the experimental data at high and low laser fluences. This also indicates that the thermal conductivity becomes low for high temperatures.

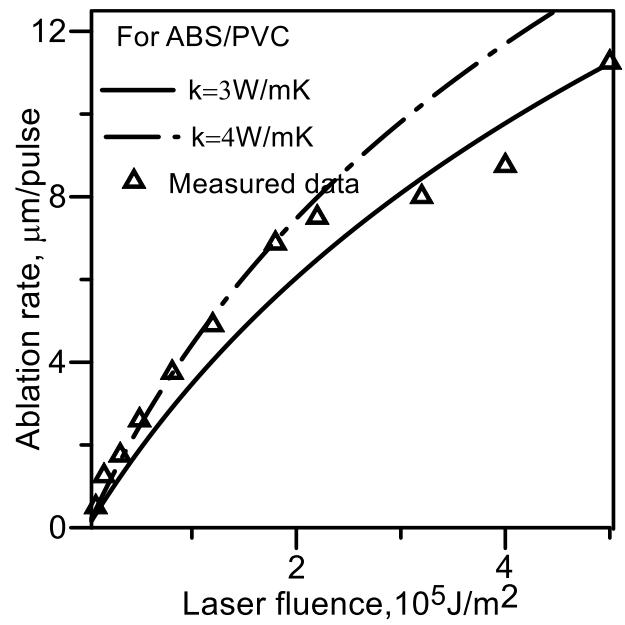

Fig. 3. Relation between ablation depth per pulse and laser fluences for different thermal conductivities

The effect of laser pulse duration on the ablation rate of ABS/PVC is shown in Fig. 4 for different laser fluences. The long irradiating time occurs for the long pulse duration of laser. This is the reason why the ablation depth per pulse increases with the increasing laser pulse duration. 


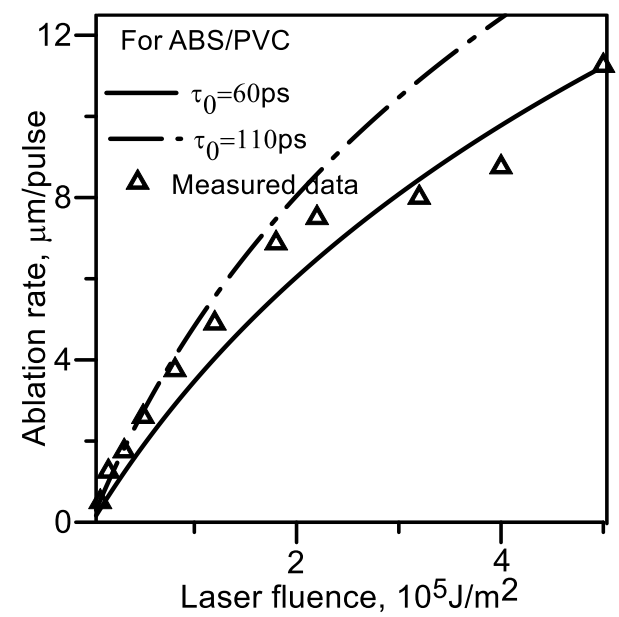

Fig. 4. Relation between ablation depth per pulse and laser fluences for different laser pulse durations

The effects of mass density and heat capacity on the variation of the ablation depth per pulse with laser fluences are, respectively, illustrated in Fig. 5 and Fig. 6. The small mass density and heat capacity give rise to the increase of the ablation rate. This is attributed to it that the smaller mass density and heat capacity can make temperature of materials rise higher for the same laser heat.

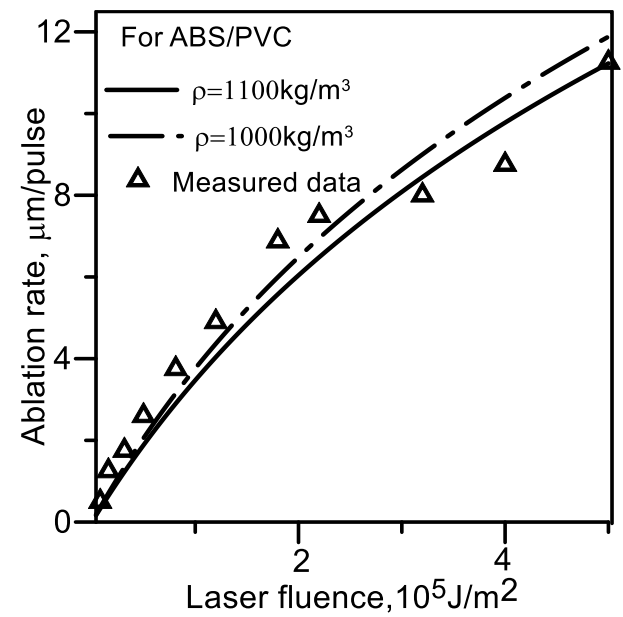

Fig. 5. Relation between ablation depth per pulse and laser fluences for different mass densities

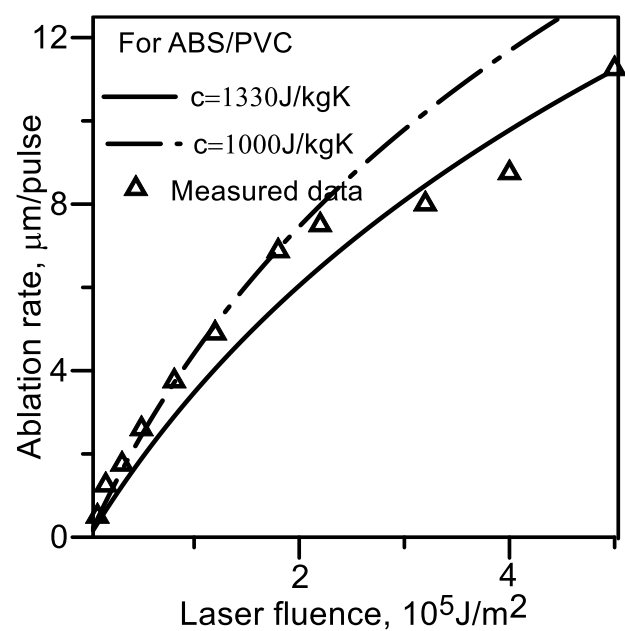

Fig. 6. Relation between ablation depth per pulse and laser fluences for different heat capacities

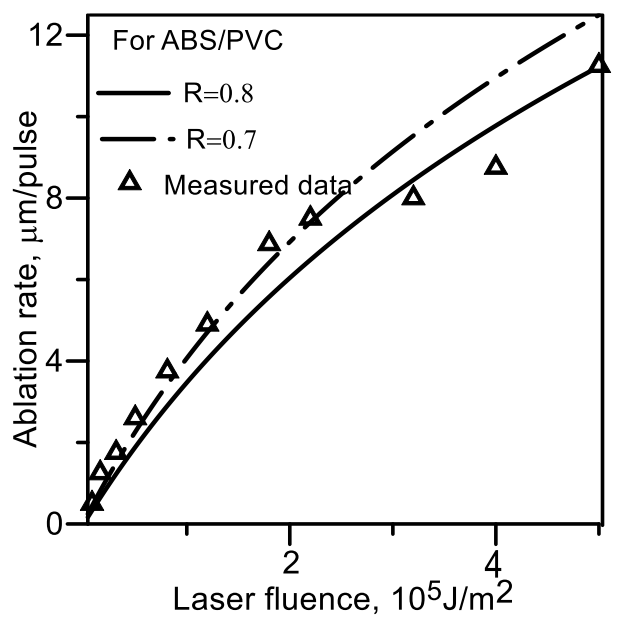

Fig. 7. Relation between ablation depth per pulse and laser fluences for different reflectivities

Fig. 7 shows the relation between the ablation depth per pulse and laser fluence for different reflectivities. The low reflectivity indicates the decrease of escaping laser light from material surface after laser light irradiates material and enhances the material absorption opportunity for incident laser optical energy. Therefore, the deeper ablation depth per pulse is obtained for the low reflectivity of material.

\section{CONCLUSIONS}

This study employs the model considering the optical absorption, thermal transport in $\mathrm{ABS} / \mathrm{PVC}$, energy balance and ABS/PVC decomposition at the ablated interface to predict the depth of laser ablation for ABS/PVC. The ablation rate predicted from this model agrees well with experimental data. This paper demonstrates that the laserpulse ablation rate at the initial short period is dominated by optical penetration absorption for laser and is linearly proportional to the logarithm of the laser fluence by a ratio factor "optical absorption length" (i.e., The ablation rate follows Beer's law). The variation of ablation depth per pulse with laser fluences are enhanced by the increasing the thermal conductivity and laser pulse duration. However, the increase of heat capacity, energy-distribution radius, reflectivity and mass density reduces the ablation depth per pulse.

\section{Acknowledgments}

This work was supported by Guangdong educational department of scientific research project (Grants No. 2017GKQNCX118) and Dongguan Polytechnic scientific research fund (Grant No. 2017a04).

\section{REFERENCES}

1. Zhong, P., Ma, S., Yang, Y., Wang, H., Liu, R., Sun, Y. Finite Element Analysis of Bulge Forming of Laser Welding Dimple Jacket Materials Science (Medziagotyra) 21 2015: pp. 626-629. http://dx.doi.org/10.5755/j01.ms.21.4.9704

2. Yang, Z., Liu, A., Yang, R. Analysis of Laser-Brazed Diamond Particle Microstructures Materials Science (Medziagotyra) 21 2015: pp. 616-621. https://doi.org/10.5755/j01.ms.21.4.9626 
3. Adomavičiūtė, E., $\quad$ Tamulevičius, T., Šimatonis, L., Fataraitè-Urbonienè, E., Stankevičius, E., Tamulevičius, S. Microstructuring of Electrospun Mats Employing Femtosecond Laser Materials Science (Medziagotyra) 21 2015: pp. 44-51. https://doi.org/10.5755/j01.ms.21.1.10249

4. Stankevičius, E., Malinauskas, M., Gedvilas, M., Voisiat, B., Račiukaitis, G. Fabrication of Periodic MicroStructures by Multi-Photon Polymerization Using the Femtosecond Laser and Four-Beam Interference Materials Science (Medziagotyra) 17 2011: pp. 244-248. https://doi.org/10.5755/j01.ms.17.3.587

5. Pique, A., $\quad$ Auyeung, R.C.Y., $\quad$ Stepnowski, J.L., Weir, D.W., Arnold, C.B., McGill, R.A., Chrisey, D.B. Laser Processing of Polymer Thin Films for Chemical Sensor Applications Surface and Coatings Technology 163-164 2003: pp. 293-299. https://doi.org/10.1016/S0257-8972(02)00606-0

6. Serafetinides, A.A., Makropoulou, M.I., Skordoulis, C., Kar, A.K. Ultra-Short Pulsed Laser Ablation of Polymers Applied surface science 180 2001: pp. 42-56. https://doi.org/10.1016/S0169-4332(01)00324-5

7. Yu, Y., Jiang, L., Cao, Q., Xia, B., Wang, Q., Lu, Y. Pump-Probe Imaging of the fs-ps-ns Dynamics during Femtosecond Laser Bessel Beam Drilling in PMMA Optics Express 23 2015: pp. 32728-32735. https://doi.org/10.1364/OE.23.032728

8. Ortiz, R., Moreno-Flores, S., Quintana, I., Vivanco, MdM., Sarasua, J.R., Toca-Herrera, J.L. UltraFast Laser Microprocessing of Medical Polymers for Cell Engineering Applications Materials Science and Engineering: C 37 2014: pp. 241-250. https://doi.org/10.1016/j.msec.2013.12.039

9. Chen, B.C., Ho, C.Y., Wen, M.Y., Chen, C.S., Tsai, Y.H., Ma, C. Ultrashort-Laser-Pulse Machining Characteristics of Aluminum Nitride and Aluminum Oxide Ceramics International 41 2015: pp. s191-s196. https://doi.org/10.1016/j.ceramint.2015.03.124

10. Chen, B.C., Tsai, Y.H., Ho, C.Y., Chen, C.S., Ma, C. Parametric Effects on Femtosecond Laser Ablation of $\mathrm{Al}_{2} \mathrm{O}_{3}$ Ceramics Ceramics International 39 2013: pp. s341-s344. https://doi.org/10.1016/j.ceramint.2012.10.090

11. Tsai, Y.H., Chen, B.C., Ho, C.Y., Chiou, Y.J., Chen, K.H., Chen, C.S., Wen, M. Y. Ablation Characteristics of Femtosecond Laser Processing for Nanometer-Sized Ceramic Films Ceramics International 43 2017: pp. s573-s577. https://doi.org/10.1016/j.ceramint.2017.05.308
12. Ho, C.Y., Chen, B.C., Chen, D.Y., Ma, C., Shih, C.S. Analytical Study on Femtosecond Laser Ablation of Aluminum Nitride Ceramics Chinese Journal of Physics 50 2012: pp. $947-955$. https://doi.org/10.6122/CJP.50.947

13. Chen, B.C., Lee, Y.C., Ho, C.Y., Wen, M.Y., Tsai, Y.H. Analysis of Removal Region in Nanoscale Metal Film Processed by Ultrafast-Pulse Laser Computational Materials Science 117 2016: pp. 590-595. https://doi.org/10.1016/j.commatsci.2015.11.037

14. Ho, C.Y., Wen, M.Y., Chen, B.C., Tsai, Y.H. Nonfourier Two-Temperature Heat Conduction Model Used to Analyze Ultrashort-Pulse Laser Processing of Nanoscale Metal Film Journal of Nanoscience and Nanotechnology 14 2014: pp. $5581-5586$. https://doi.org/10.1166/jnn.2014.9516

15. Ho, C.Y., Tsai, Y.H., Chen, B.C. Investigation into Pulse Laser Heating of Nanoscale Au Film Using Dual-Phase-Lag Model Journal of Nanoscience and Nanotechnology 13 2013: pp. $7205-7207$. https://doi.org/10.1166/jnn.2013.7947

16. Ho, C.Y., Chen, B.C., Yu, J.W., Tsai, Y.H., Wen, M.Y. Femtosecond Laser Ablating Depth for Nanometer-sized Thin Metal Films Journal of Nanoscience and Nanotechnology 17 2017: pp. 5893-5895. https://doi.org/10.1166/jnn.2017.14157

17. Ho, C.Y., Shih, C.S., Hung, K.M., Ma, C., Lin, S.Y. A Model for Femtosecond-Laser-Pulse Ablation of Metal Thin Films Chinese Journal of Physics 50 2012: pp. 939-946. https://doi.org/10.6122/CJP.50.939

18. Bityurin, N.M. Thermal Volume Model for Laser Ablation of Polymers Izvestiya Akademii Nauk Seriya Fizicheskaya 65 2001: pp. 532-535. (in Russian) https://doi.org/10.1021/cr010426b

19. Gedvilas, M., Raciukaitis, G. Investigation of UV Picosecond Laser Ablation of Polymers Proceedings SPIE: Workshop on Laser Applications in Europe 6157 2005: pp. 61570T-1-10. https://doi.org/10.1117/12.661141 